\title{
Expression Divergence as an Evolutionary Alternative Mechanism Adopted by Two Rice Subspecies Against Rice Blast Infection
}

\author{
Zhenhui Zhong ${ }^{1,2+}$, Lianyu Lin ${ }^{1,2,3+}{ }^{+}$, Meilian Chen ${ }^{1,2}$, Lili Lin ${ }^{1,2}$, Xiaofeng Chen ${ }^{1,2}$, Yahong Lin ${ }^{1,2}$, Xi Chen ${ }^{1,2}$, \\ Zonghua Wang ${ }^{1,2,4}$, Justice Norvienyeku ${ }^{1,2^{*}}$ and Huakun Zheng ${ }^{1,2^{*}}$ (D)
}

\begin{abstract}
Background: Rice (Oryza sativa L.) is one of the most important crops that serves as staple food for $\sim 50 \%$ of the human population worldwide. Some important agronomic traits that allow rice to cope with numerous abiotic and biotic stresses have been selected and fixed during domestication. Knowledge on how expression divergence of genes gradually contributes to phenotypic differentiation in response to biotic stress and their contribution to rice population speciation is still limited.

Results: Here, we explored gene expression divergence between a japonica rice cultivar Nipponbare and an indica rice cultivar 93-11 in response to invasion by the filamentous ascomycete fungus Magnaporthe oryzae (Pyricularia oryzae), a plant pathogen that causes significant loss to rice production worldwide. We investigated differentially expressed genes in the two cultivars and observed that evolutionarily conserved orthologous genes showed highly variable expression patterns under rice blast infection. Analysis of promoter region of these differentially expressed orthologous genes revealed the existence of cis-regulatory elements associated with the differentiated expression pattern of these genes in the two rice cultivars. Further comparison of these regions in global rice population indicated their fixation and close relationship with rice population divergence.

Conclusion: We proposed that variation in the expression patterns of these orthologous genes mediated by cis-regulatory elements in the two rice cultivars, may constitute an alternative evolutionary mechanism that distinguishes these two genetically and ecologically divergent rice cultivars in response to M. oryzae infection.
\end{abstract}

Keywords: Expression divergence, Rice blast disease, Pathogen response, Rice divergence

\section{Background}

Expressional divergence mediated by variation in cis-elements has long been considered as an important force in phenotypic evolution (Siepel and Arbiza 2014). Generally, cis-elements are localized upstream of the coding sequence and manipulate the expression pattern of genes by altering the binding of transcription factors required for gene expression (Wray 2007). Accumulated data indicated that changes in cis-elements could lead to phenotypic variation and consequently cause phenotypic evolution within or

\footnotetext{
* Correspondence: jk_norvienyeku@fafu.edu.cn; huakunzheng@163.com 'Zhenhui Zhong and Lianyu Lin contributed equally to this work.

${ }^{1}$ State Key Laboratory of Ecological Pest Control for Fujian and Taiwan Crops, College of Plant Protection, Fujian Agriculture and Forestry University,

Fuzhou 350002, China

Full list of author information is available at the end of the article
}

between species. For example, the homologous proteins in humans and chimpanzees share more than $99.5 \%$ identity, but the two species differ from each other in many phenotypes (King and Wilson 1975), which was later turned out to be the consequences of divergent expression caused by variation in cis-elements associated with their coding genes (Siepel and Arbiza 2014). Similar mechanism was also identified in other species, such as Arabidopsis (Arsovski et al. 2015), sorghum (Jiang et al. 2013) and Drosophila (Osada et al. 2006; Rifkin et al. 2003). However, most of these studies were focused on the developmental phenotype, and how does the cis-elements mediated expressional divergence contributes to phenotypic differentiation in response to diverse stresses remains largely unknown. 
Rice (Oryza sativa L.) is one of the most important crops that serves as staple food for more than half of the world's population. Over the years, rice has evolved into several subspecies as a result of purposeful breeding and intensive domestication. These different rice subspecies are often endowed with distinct agronomic traits, such as larger grain size, superior grain quality as well as biotic and abiotic stress tolerance (Wang et al. 1998). The speciation of rice species is greatly influenced by human activities. Recent rice population genetic studies demonstrated that the two most widely distributed rice subspecies japonica Group and indica Group evolved from a common ancestor, Oryza rufipogon several thousand years ago (Huang et al. 2012, Wang et al. 2018b, Xie et al. 2015). Additional rice population studies further showed that environmental factors, especially temperature, exerted great influence on the genomic characteristics of japonica and indica rice cultivars (Huang et al. 2012). Many of the domestication related genes have been identified as key regulators of important agronomic traits, such as seed shattering, pericarp color, amylose content, as well as grain number or size in rice, and these genes have shown significant variation between rice subspecies. For instance, a major shattering QTL, qSH1, has been identified in all temperate japonica rice but absent in tropical japonica and indica rice (Konishi et al. 2006). Some differentially expressed genes in response to various signals or different life stages which lead to distinct features have been fixed during the domestication history of rice (Jung et al. 2008; Liu et al. 2010a; Lu et al. 2010). Recent studies revealed SNPs or InDels at promoter regions between rice subspecies that leads to dramatic phenotypic differentiation in rice blast resistance, grain yield and nitrogen utilization (Bai et al. 2017; Huang et al. 2018; Li et al. 2017; Wang et al. 2018a). However, the origin and genomic foundations related with SNPs or InDels mediated variation on gene expression still need to be uncovered.

In an attempt to control the development of rice blast disease, caused by the filamentous ascomycete fungus Magnaporthe oryzae (Pyricularia oryzae), which causes significant yield losses in rice producing regions across the world, hundreds of resistance related genes have been domesticated along with human rice breeding history (Dean et al. 2012; Ebbole 2007; Li et al. 2017; Liu et al. 2010b). Similar to other distinctly manipulated agronomic traits, japonica and indica cultivars exhibited different degrees of resistance against $M$. oryzae (Bhuiyan et al. 2007; Gallet et al. 2016). Our recent results implied japonica and indica cultivation may shape population divergence of $M$. oryze (Zhong et al. 2018). However, it's still not clear how expression level variation of rice blast infection responsive genes shapes the population speciation of rice.
Here, we profiled a japonica Group cultivar (Nipponbare, NPB) and an indica Group cultivar (93-11) in response to the fungal pathogen infection, by mainly focusing on the performance of orthologous genes. Our analysis showed that the distinct expression patterns exhibited by orthologous genes identified in the two rice cultivars corresponded with the high sequence diversity prevailing in the promoter region. Additionally, we further investigated genetic divergence of identified sequences in 3,010 rice cultivars and found that these regions exhibited higher fixation index (Fst) between japonica and indica rice populations, which indicates that these regions undergone strong selection during rice population divergence.

\section{Methods}

\section{Rice Cultivation and Inoculation}

Magnaporthe oryzae isolates Guy11 and FJ81278 were used for inoculating rice. The conidia harvested from 10-day-old colonies were cultured on rice-bran agar medium ( $2 \%$ rice-polish, $1.5 \%$ agar, and $\mathrm{pH} 6.5$ ) at $26^{\circ} \mathrm{C}$ under constant light. Conidia suspensions were adjusted to $1.5-2.0 \times 10^{5}$ conidia $/ \mathrm{mL}$ in solution containing $0.02 \%$ Tween before using it to inoculate four-week-old rice seedlings (Oryza sativa L.) cultivar Nipponbare (NPB, japonica) and 93-11 (indica) along with control respective groups (rice seedlings sprayed with solution containing only $0.02 \%$ Tween). To avoid the influence of dark treatment after inoculation, both inoculated rice and control groups were incubated in the dark, humid chamber at $25^{\circ} \mathrm{C}$ for $24 \mathrm{~h}$ before transferring them into another humid chamber with $12 \mathrm{~h}$ photoperiod or sample collection. Plant tissues were collected at 24 hpi (collect both mock samples and samples inoculated by isolates for $24 \mathrm{~h}$ ) and $48 \mathrm{hpi}$. All the samples were immediately frozen in liquid nitrogen after collection and stored at $-80^{\circ} \mathrm{C}$. The experimental samples were generated from three biological repeats.

\section{RNA Extraction, Sequencing Library Construction and Illumina Sequencing}

Total RNA was extracted with RNAprep pure Plant Kit (Tiangen, Beijing) according to the manufacturer's instructions. RNA integrity and quantity was verified using a 2100 Bioanalyzer (Agilent Technologies, Santa Clara, CA) according to the manufacturer's instructions. The cDNA library was sequenced using the Illumina sequencing platform (IlluminaHiSeq ${ }^{\text {Tm }}$ 2000) with $125 \mathrm{bp}$ pair-end reads length and $280 \mathrm{bp}$ insert size by Gene Denovo Co. (Guangzhou, China). An in-house Perl program was used to select clean reads by removing adaptor sequences, low quality sequences (reads with more than $50 \%$ of base quality lower than 20) and reads with more than $5 \% \mathrm{~N}$ bases. 
Reads Alignment and Normalization of Gene Expression Levels

The reference genome for RNA seq analysis is Nipponbare genome (Oryza sativa japonica: http://plants.ensembl.org/Oryza_sativa/Info/Index). Cleaned short reads were aligned to all exon sequence by Bowtie2 (Langmead and Salzberg 2012), and expression abundance were calculated by RSEM with default parameters (Li and Dewey 2011). Gene expression level obtained from RNA-Seq analysis was validated by qRT-PCR for 10 DEGs. Primers used in the study were as shown in Additional file 1: Table S2.

\section{Differentially Expressed Genes (DEGs) and Function Enrichment Analyses}

To reduce false positive of differential expression, all isoforms with less than 200 reads count in total were regarded as low-expressed genes and have been removed in the following analysis. Differential expression analysis was conducted with edgeR (Robinson et al. 2010). False discovery rate (FDR) was used to control the $P$-value and a threshold of $\mathrm{FDR}<0.05$ and $|\log 2 \mathrm{FC}|>1$ was used in determining significant differences in the expression level between samples. Gene Ontology Consortium (http://geneontology.org/) was used for functional enrichment analysis of differentially expressed genes (Ashburner et al. 2000).

\section{Identification of Orthologous Genes, Collinearity Analysis and Motif Enrichment}

Orthologous genes from NPB and 93-11 were identified by NCBI-BLASTN with 500 xdrop-gap parameters. To reduce the redundancy of orthologous genes obtained from BLASTN, $2 \mathrm{~kb}$ upstream sequence was also included for searching the best hit that shares the most similar coding and flanking sequence. MCScanX was used to identify the collinearity between NPB and 93-11 with default parameters (Wang et al. 2012). Genome collinearity was visualized with Circos (Krzywinski et al. 2009). To analyze orthologous genes in other rice genome, we have searched orthologous genes in 3 indica rice (Zhenshan 97, Minghui 63 (Zhang et al. 2016), Shuhui 498 (Du et al. 2017)) and 2 japonica rice (HEG4, A123 (Lu et al. 2017)) with NCBI-BLASTN.

Motif enrichment was performed by using the HOMER findMotifs tool with default parameters (Heinz et al. 2010). Selection of input sequences and background sequences for motif enrichment are as shown in Fig. $3 \mathrm{~b}$ and $\mathrm{c}$.

\section{Nucleotide Diversity Calculation and Population Genomic Data Analysis}

$2 \mathrm{~kb}$ upstream sequence of orthologs was extracted and Needle was used for pair-wise sequence alignment (Rice et al. 2000). DnaSP was used for nucleotide diversity calculation (Rozas et al. 2017). SNPs data of 1,770 indica and 850 japonica rice were obtained from Rice SNP-Seek Database (http://snp-seek.irri.org/) for population genomics analysis (Wang et al. 2018b). Samtools and Vcftools was used to calculate the fixation index (Fst) of different orthologs (Danecek et al. 2011; Li et al. 2009).

\section{Results}

RNA-Seq Sequencing of NPB and 93-11 in Response to $M$. oryzae Infection

To investigate the expression divergence between japonica and indica rice groups under $M$. oryzae infection, we performed comparative transcriptome analysis between NPB and 93-11 challenged with two $M$. oryzae strains, Guy11 and FJ81278, respectively. NPB and 93-11 were used as representative rice cultivars of japonica and indica, respectively, in this study, since the genome sequences of these two rice cultivars are well described in previous studies (Goff et al. 2002; Yu et al. 2002). While FJ81278 (FJ87) belongs to the indica-infecting lineage (clade3), Guy11 (GY11) belongs to a lineage that consisted both the indica-infecting and japonica-infecting population (Bao et al. 2017; Zhong et al. 2018). Infection assay of NPB/93-11 with GY11/FJ87 showed that both isolates can cause susceptible reaction on the two rice cultivars with slightly different aggressiveness at 18, 24, 36 and $48 \mathrm{~h}$ post inoculation (hpi) (Additional file 1: Figure S1). The workflow portraying sample inoculation and collection is shown in Additional file 1: Figure S2. Rice samples treated with or without $M$. oryzae spore solutions were collected at $24 \mathrm{hpi}$ and $48 \mathrm{hpi}$ respectively. In total, 402 million and 434 million of $125 \mathrm{bp}$ paired-end reads for NPB and 9311 were generated with three biological replicates. Cleaned sequence reads for 30 samples were mapped against reference genome sequence for gene expression calculation (Additional file 1: Table S1). Differentially expressed genes (DEGs) were identified according to their significance in expression fold-change (false discovery rate (FDR) $<0.05$ and at least two-fold change $(|\log 2 \mathrm{FC}|>1)$ in comparison to their respective control. The Pearson Correlation Coefficient of three biological replicates at three time points of rice cultivars infected by the two isolates are 0.96 (NPB) to 0.97 (93-11) (Additional file 1: Figure S3). We further validated the dataset obtained from RNA-seq with qRT-PCR by choosing 10 DEGs from NPB and 93-11 respectively. The correlation $\left(\mathrm{R}^{2}\right)$ of RNA-seq and qRT-PCR results are 0.80 and 0.83 for NPB and 93-11 respectively, demonstrating high reliability of the sequencing results (Additional file 1: Figure S4). We also analyzed expression level of some important defense genes include WRKY80, PR1a, PR1b, PR3, PR5, PR10, LOX, AOS, PAL and CHS1 in our RNA-seq data, and confirmed their up-regulation in our data (Additional file 1: Figure S5). 
Together, these results indicate high reproducibility of sequencing results.

\section{Comparative Expression Analysis of NPB and 93-11 Under M. oryzae Infection}

A total of 27,844 (NPB) and 25,962 (93-11) genes were obtained and deployed for differential expression analysis after filtering-out lowly expressed genes. A total of 8,948 and 8,384 differentially expressed genes (DEGs) were identified in NPB and 93-11 by comparing between each other at different time points (Fig. 1a). As shown in Fig. 1a, with increased time of infection, the number of DEGs increased between mock control with 24 and 48 hpi in two rice cultivars, indicating more genes have been suppressed or activated during $M$. oryzae colonization. Moreover, though GY11 and FJ87 only exhibited slightly different aggressiveness on rice infection process, they can induce different response patterns on the two rice cultivars. For instance, we found that more DEGs of FJ87 infected rice than GY11 infected rice at $24 \mathrm{hpi}$, while more DEGs of GY11 infected rice than FJ87 infected rice at $48 \mathrm{hpi}$. In addition, we found that more DEGs between 24hpi and 48hpi in FJ87 inoculated NPB or 93-11 than GY11. It's possible that these two isolates shown different fitness on two rice cultivars because these two isolates from distinct lineages, as we identified through large scale population genomic study, have some isolate-specific effectors that help pathogen manipulate plant early immunity activate different response genes in plant (Liao et al. 2016; Zhong et al. 2018).

To understand the response mechanisms to $M$. oryzae in two rice cultivars, we conducted Gene Ontology (GO) enrichment analysis of response genes in two rice cultivars. GO enrichment analysis revealed that numerous pathogen responsive pathways were activated during $M$. oryzae infection (Fig. 1b). For instance, diterpene phytoalexin biosynthetic pathway and chorismate biosynthetic pathway which have been shown to be activated in response to pathogen attack in various plants were enriched in DEGs carried-out in our study (Rao and Strange 1994; Wildermuth et al. 2001). In addition, we noticed genes belong to different GO term were enriched in two rice cultivars differently. For instance, trehalose metabolism, cinnamic acid processing and lysine biosynthetic process genes were enriched at NPB, while glycine metabolic process, regulation of jasmonic acid mediated signaling pathway and leucine metabolic process genes were enriched in 93-11, which suggest two rice cultivars may use different set of genes in response to M. oryzae infection (Bowles 1990; Schaff et al. 1995; Yang and Ludewig 2014; Zeier 2013).

\section{Differential Characteristics of Orthologous Genes in NPB and 93-11 in Response to Infection}

NPB and 93-11 belong to japonica group and indica group respectively. These two rice groups divided several thousand years ago, which may have brought lots of differentiation in the genome (Sweeney and McCouch 2007). To determine the extent of divergence between two rice genomes, we compared genomic collinearity between the two cultivars. From this analysis, we observed the existence of high inter-genomic collinearity between the two cultivars. We also observed that, $\sim 97.3 \%$ $(13,501 / 13,876)$ of the orthologous genes showed high inter-genomic collinearity between NPB and 93-11. In addition, we further analyzed 13,876 of orthologous genes in 5 rice genomes ( 2 japonica and 3 indica rice), and found that these orthologous genes are also very conserved in these genomes (Additional file 1: Figure S6).

To investigate whether NPB and 93-11 used different sets of genes in response to $M$. oryzae infection, we divided
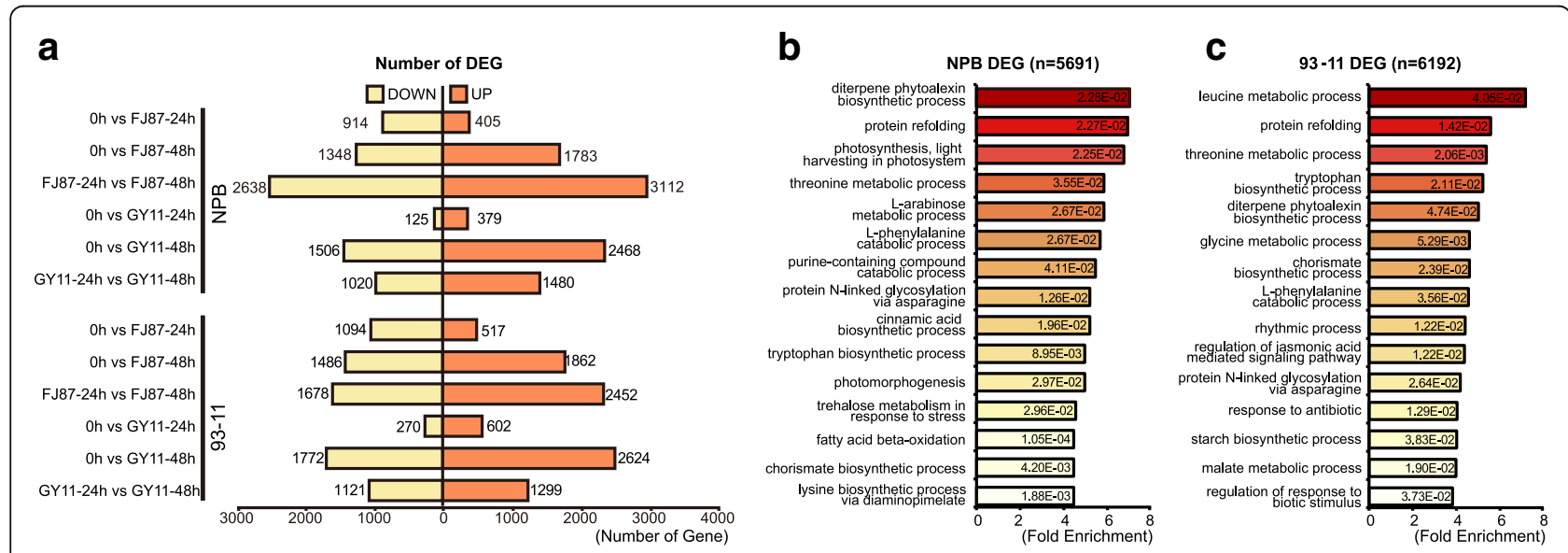

Fig. 1 Number and GO enrichment of DEGs. a Up and down regulated genes in NPB and 93-11 between different time point and isolates. DOWN: down regulated genes. UP: up regulated genes. DEGs were defined as false discovery rate (FDR) $<0.05$ and at least two-fold change $(|\log 2 \mathrm{FC}|>1)$. b and $\mathbf{c}$ Gene ontology $(\mathrm{GO})$ enrichment of all differential expressed genes of NPB and 93-11. Fold Enrichment was calculated by observed gene number divided by expected gene number in each $\mathrm{GO}$ term. $P$ value was calculated by Fisher's Exact with Bonferroni correction. 
whole genome genes into 13,876 pairs of orthologous genes and 33,327 non-orthologous genes for further comparison. Interestingly, our studies showed that orthologous genes, which are considered as genetically conserved region in evolution, displayed differential expression profile in these two cultivars under infection (Table 1), indicating the existence of strong selection pressure on expression level of orthologous genes in the course of intensive domestication of japonica and indica rice subspecies as an evolutionary tool for resisting $M$. oryzae infection. Our investigations showed that a total of 3,491 (25.2\%) and 3,526 (25.4\%) of orthologous genes that exhibited differential expression pattern in the two rice cultivars at $24 \mathrm{hpi}$ or $48 \mathrm{hpi}$, which is significantly higher than $19.4 \%$ and $17.4 \%$ of non-orthologs in NPB and 93-11 respectively (Table 1, two-sided Fisher's exact test, $p$-value $=2.26 \mathrm{e}^{-42}(\mathrm{NPB})$ and $\mathrm{p}$-value $=2.73 \mathrm{e}^{-85}$ (93-11)). By comparing orthologous DEGs number in two rice against GY11 or FJ87 at different time points, we found the number of DEGs in both NPB and 93-11 are much lower than DEGs at only one rice cultivar (either in NPB or 93-11) (Fig. 2b and c). To investigate overlapping of orthologous DEGs in the two rice, we merged DEGs at different time points or against different isolates and tested overlap between the two rice. As shown in the Venn diagram, $36.99 \%(1,291 / 3,491$ for NPB) and $37.61 \%$ (1,326/3,526 for 93-11) of orthologous genes were differentially expressed in NPB and 93-11 at 24 hpi or 48 hpi of M. oryzae infection (Fig. 2d). From these results we inferred that, conserved orthologous genes identified in the two cultivars evolved independently from each other and rice blast pathogens evolved different strategies to adapt to the host basal resistance.

\section{Variation in Promoter Region May Account for Divergence in the Expression Pattern of Orthologous Genes}

Although orthologous genes showed high genomic collinearity and sequence similarity (>95\%), they however responded differently to biotic stress induced by $M$. oryzae infection. We speculated promoter regions, where the cis-regulatory elements are enriched, may have experienced significant changes. To compare variation level of promoter regions, we divided orthologous genes into NPB and 93-11 common DEGs (common DEG), NPB specific DEGs (NPB unique DEG), 93-11 specific DEGs (93-11 unique DEG) and the rest that not DEG in NPB or 93-11
(Non DEG), and compared their nucleotide diversity in both promoter regions and coding sequence (CDS). Since the sequence similarity level of orthologous genes in two rice will greatly affect sequence alignment as well as nucleotide diversity calculation, we decided to use more stringent methods to filter out less conserved orthologous genes. To achieve this, only genes with similar expression level and high sequence similarity between two rice were defined as common DEGs. Consistently, only genes that have differential expression level between two cultivars and high sequence similarity were defined as NPB/93-11 unique DEGs. Finally, 547 and 629 pairs of unique DEGs of NPB and 93-11 as well as 1,222 pairs of common DEGs, 9,095 pairs of non DEGs were identified. By comparing nucleotide diversity of these four groups, we found that nucleotide diversity in promoter regions is generally higher than that of CDS and as expected, NPB/93-11 unique DEGs exhibited significantly higher nucleotide diversity than common DEGs or Non DEGs (Student's $t$-test, $p$-value equal to $7.89 \mathrm{e}^{-05}$ in $93-11$ unique DEGs group and $1.21 \mathrm{e}^{-05}$ in NPB unique DEGs group). As shown in Fig. 3a, average level of nucleotide diversity for 93-11 unique DEGs, NPB unique DEGs, common DEGs and non DEGs at promoter regions are 0.0136, 0.0144, 0.0106 and 0.0121 , compared with $0.003,0.004,0.002$ and 0.003 at CDS regions respectively. In addition, average level of nucleotide diversity for 93-11 unique DEGs, NPB unique DEGs are significantly higher than that of common DEGs and non DEGs at promoter regions $(0.0136$ and 0.0144 vs. 0.0106 and 0.0121 ).

NPB unique DEGs or 93-11 unique DEGs are genes that specifically and differentially expressed in NPB or 93-11, which may be mediated by variation at promoter region. To investigate whether variation at promoter regions results in cis-regulatory elements variation, we set out to conduct cis-regulatory elements enrichment of these regions. To achieve this, we collected sequences at promoter regions with SNPs or InDel between NPB and 93-11 and its $25 \mathrm{bp}$ up/down flanking sequences and applied to motif enrichment analysis with the same regions of orthologs at opposite rice cultivar as background control (Fig. 3b). From this analysis, we have identified 29,921 SNPs and 10,135 InDels at promoter regions of $\mathrm{NPB} / 93-11$ unique DEGs in the two rice cultivars. Motif enrichment analysis indicated that promoter sequences variation lead to cis-regulatory

Table 1 Rates of orthologous DEGs and non-orthologous DEGs in 93-11 and NPB in response to M. oryzae infection

\begin{tabular}{|c|c|c|c|c|c|c|c|c|}
\hline & \multicolumn{4}{|l|}{ NPB } & \multicolumn{4}{|l|}{$93-11$} \\
\hline & Expressed & Rate & DEGs & Rate & Expressed & Rate & DEGS & Rate \\
\hline Ortholog & 10,337 & $74.5 \%$ & 3,491 & $25.2 \%$ & 10,142 & $73.1 \%$ & 3,526 & $25.4 \%$ \\
\hline Non-Ortholog & 17,507 & $62.0 \%$ & 5,485 & $19.4 \%$ & 15,820 & $56.0 \%$ & 4,907 & $17.4 \%$ \\
\hline Total & 27,844 & $66.1 \%$ & 8,948 & $21.2 \%$ & 25,962 & $61.6 \%$ & 8,384 & $19.9 \%$ \\
\hline
\end{tabular}



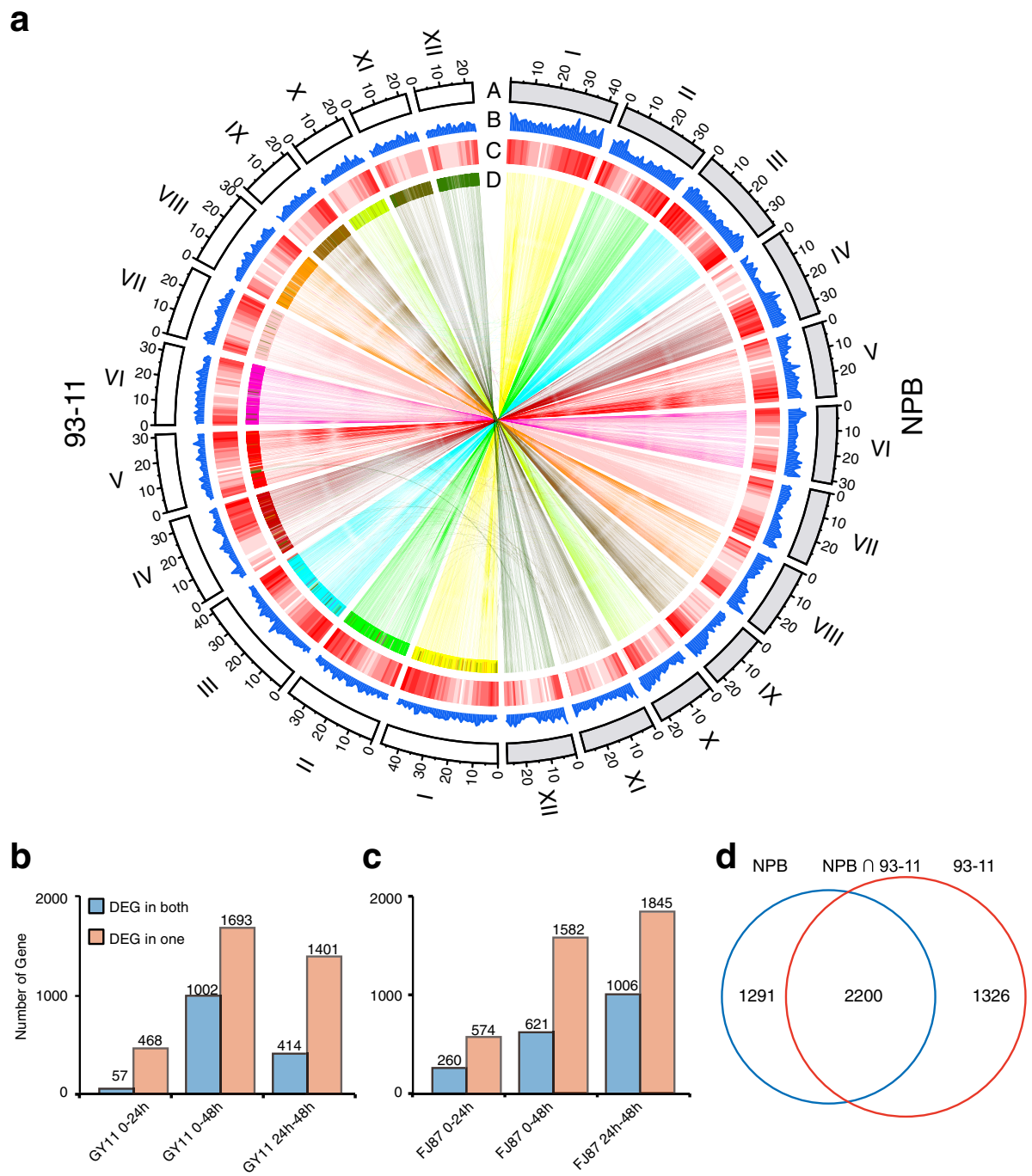

Fig. 2 Different performance of orthologous genes in response to M. oryzae infection. a Circos map was used to describe whole genome composition, distribution of genes and collinearity of orthologous genes. Track a: 12 chromosomes of NPB and 93-11. Track b: numbers of unique genes in a $1 \mathrm{Mb}$ window. Track c: density of orthologous genes over genome, white to red gradient color stands for dense and disperse regions respectively. Track d: collinearity of orthologous genes between two rice cultivars. b Number of orthologous DEGs in NPB and 93-11 in response to GY11 infection between different time points. c Number of orthologous DEGs in NPB and 93-11 in response to FJ87 infection between different time points. $\mathbf{d}$ Venn graph of DEGs orthologous genes. NPB: unique DEGs of NPB. 93-11: unique DEGs of 93-11. NPB $\cap$ 93-11: common DEGs of NPB and 93-11

elements variation. Figure $3 \mathrm{c}$ listed out top 10 most enriched transcription factor binding motif in NPB and 93-11. Among these cis-regulatory elements variation, NFY, PCF, BHLH112, a C2H2 TF and SEP3 are of the most enriched transcription factor binding motif in NPB. While MED-1, Pp_0237, Trihelix, CDC5 and AGL42 binding site are the most abundant transcription factor binding motif identified in promoter regions of 93-11 unique DEGs.

\section{Variation in Promoter Regions is Associated with Rice Divergence}

After several thousand years of domestication, japonica and indica rice constitutes two largest rice subspecies in cultivated rice, during which most important traits such as seed dormancy, shattering, seeds number, seed size and resistance have been fixed in the population (Sweeney and McCouch 2007). Since rice blast disease is one of the major biotic stress to rice, we hypothesized traits that relate to $M$. oryzae response also experienced strong selection during rice domestication. Thus, our next question is to ask whether the expression divergence mediated by cis-elements variation is also involved in the population divergence of rice japonica and indica groups. To test this hypothesis, we analyzed fixation index (Fst) of identified variation sites in population data of previously published 1,770 indica and 850 japonica 

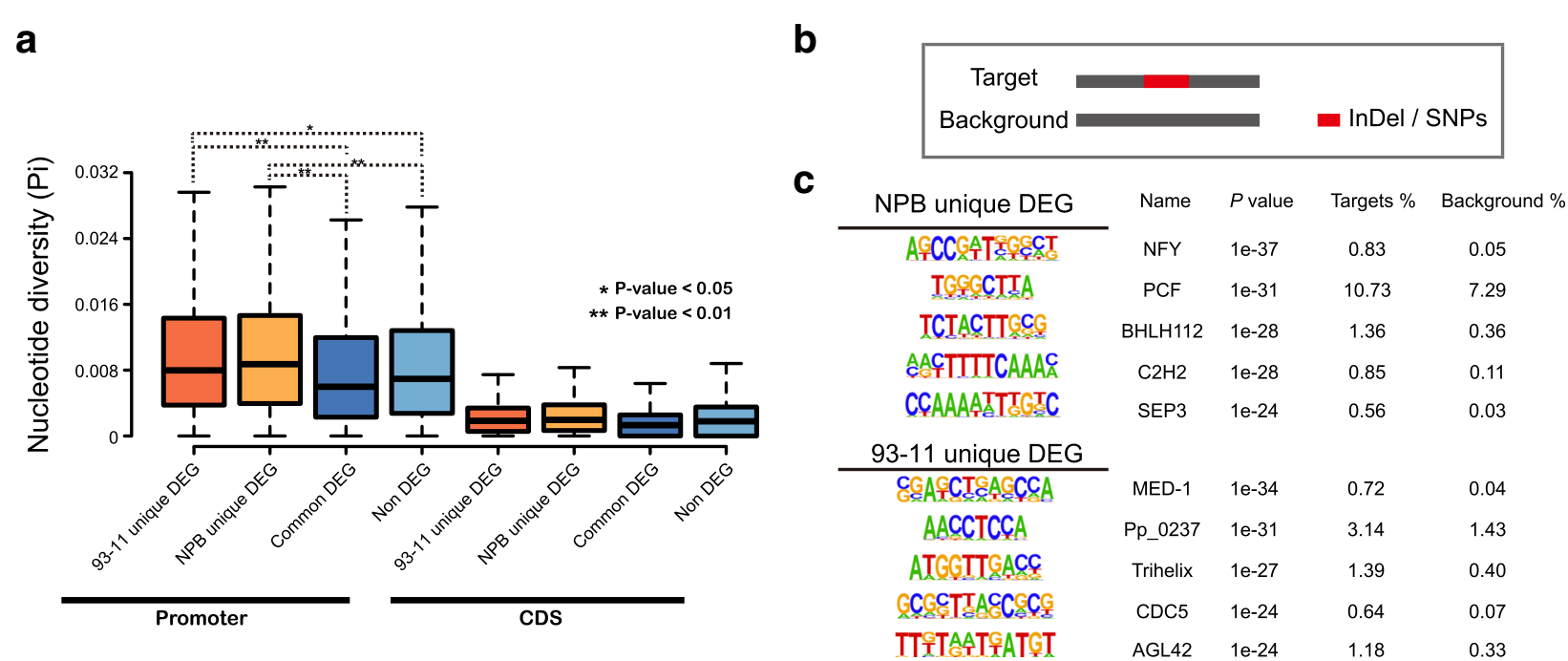

Fig. 3 High differentiation at promoter region. a Boxplot showing nucleotide diversity (Pi) of 93-11 unique DEGs, NPB unique DEGs, common DEGs and Non DEGs at promoter and CDS regions. * represents $p$-value smaller than $0.05,{ }^{* *}$ represents $p$-value smaller than 0.01 estimated with student's t-test. $\mathbf{b}$ Schematic of sequences used in motif enrichment analysis. Target sequences are sequences that have an InDels or SNPs with $25 \mathrm{bp}$ up/down-stream flanking sequence. Background sequences are corresponding site at opposite rice that have no InDels or SNPs but only 25 bp up/down-stream flanking sequence. c Top 10 enriched transcription factor binding motif in promoter regions that have InDels or SNPs in NPB or $93-11$

rice accessions, which represents global japonica and indica rice cultivars. In consistent with former study, the average Fst level of selected sites between japonica and indica groups is 0.526 (Fig. 4a), which indicate a strong differentiation between two rice subspecies (Huang et al.
2010). However, Fst value at promoter regions containing cis-elements variation in NPB and 93-11 unique DEGs are 0.547 and 0.541 respectively, which is significantly higher than that of common DEG $(F s t=0.518)$ and non DEGs $(F s t=0.523)$. In line with phylogenetic

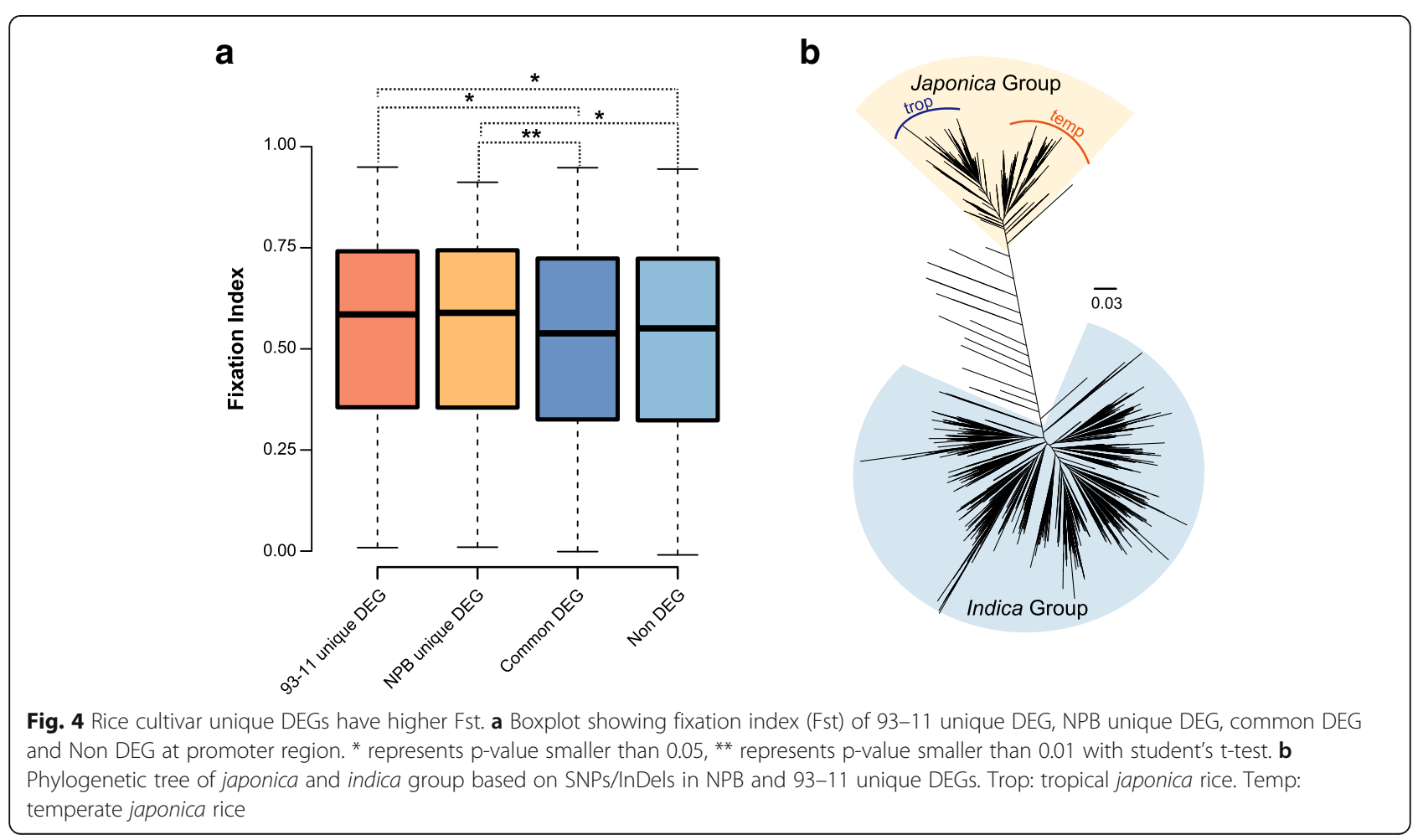


tree based on whole genome SNPs data in previous study, the phylogenetic tree based on SNPs/InDels in NPB and 93-11 unique DEGs also showed that japonica and indica rice groups could be divided into two welldefined clades (Fig. 4b), in which japonica group can be further defined as tropical japonica rice (trop) and temperate japonica rice (temp) (Wang et al. 2018b).

\section{Discussion}

Interplay between environmental factors and diverse genomic features enable various rice cultivars to possess firm resistance against invading pathogenic microbes and also equip them with the requisite traits to thrive under harsh conditions (López-Arredondo et al. 2015). Although researches have shown that different rice subspecies exhibit differential resistance against the blast fungus M. oryzae (Gallet et al. 2016), details regarding genomic parameters accounting for the differential response of different rice subspecies to blast infection has not been extensively studied. This study provides an insight into how variation in the promoter region of orthologous genes contribute to the differential response of two rice cultivars, NPB and 93-11 each from japonica and indica subspecies during M. oryzae infection. The short divergence time between japonica and indica rice as well as high quality genome sequences makes these two rice cultivars good models in solving molecular, genetic and evolutionary questions (He et al. 2011; Liao et al. 2016; Tang et al. 2006). We identified 13,876 pairs of orthologous between NPB and 93-11 genomes, and most of these genes exhibit similar or identical chromosome location in the genomic collinear analysis. However, our comparison revealed significant difference in the expression of orthologous genes identified in the two cultivars during $M$. oryzae infection. Since orthologous genes are considered as highly conserved throughout divergence history of the two japonica and indica rice cultivars (Sweeney and McCouch 2007), we opine that the observed emerging divergence in the expression pattern of orthologous genes between NPB and 93-11 rice cultivars challenged with $M$. oryzae could be an individually acquired evolutionary adaptation developed by the two rice cultivars in an attempt to durably resist rice blast fungus.

It was proposed that gene expression divergence related with adaptive phenotypes may lead to speciation (Haerty and Singh 2006). With the development of next generation sequencing, accumulating evidences have been reported showing expression divergence of orthologous genes between closely related species in response to various stresses. Expression divergence between pine and spruce, which divergent $\sim 140$ million years ago, is important for survival in the face of climatic change (Yeaman et al. 2014), and this phenomenon is also allowing domesticated and wild tomato tackle wound stress (Liu et al. 2018). In our study, we observed high incidence of variation in promoter region of orthologous genes that are known to be enriched with gene regulatory elements. We deduced that inter-cultivar variations observed in the expression pattern of orthologous genes are as a result of nucleotide diversities associated with the promoter region sequences of orthologous genes from the two cultivars. This hypothesis is supported by previous researches which showed that mutation in the cis-elements of individual promoter could cause phenotypic variation (Hoffmann et al. 2016; Xing et al. 2008). Furthermore, our studies also identified genome-wide correlation between variations occurring in the promoter region of $M$. oryzae infection responsive genes and their transcriptional divergence between NPB and 93-11.

Extensive studies indicated that sequence variation in promoter regions of cis-regulatory elements affects the specificity or binding activity of Transcription Factor (TF), which subsequently affects expression pattern of its proximal genes and resulted in obvious phenotypic changes. For instance, a single SNP at $b s r-d 1$ promoter enhances binding to MYBS1 and promote broad-spectrum rice blast disease resistance in rice (Li et al. 2017). Small insertions in ARE1 promoter in indica and aus accessions enhance grain yield under nitrogen-limiting conditions (Wang et al. 2018a). We inferred that, high variation in promoter region may trigger difference in the binding efficiency of TFs and hence, resulting in expression divergence of infection responsive genes in the two rice cultivars investigated in this study (Tran et al. 2004; Yoshida et al. 1998). Consistent with this hypothesis, we found TF binding motif of NFY CCAAT box, which is important for drought response in maize (Nelson et al. 2007), were enriched specifically at promoter regions variation sites of NPB unique DEGs. TF binding motif of PCF (also named TEOSINTEBRANCHED1, TCP), which plays key roles in plant systemic acquired resistance, was also enriched among promoter of NPB unique DEGs (Li et al. 2018). BHLH112 is characterized as abiotic stress tolerance in Arabidopsis (Liu et al. 2015). In addition, GATA factor-related transcription factors MED-1, CDC5 and AGL42 enriched at promoter variation sites of $93-11$ unique DEGs are also reported to be involved in development and immunity (Broitman-Maduro et al. 2005; Nawy et al. 2005; Palma et al. 2007). The population genomic analysis in our study further indicated that variations that affected TF binding activity may subsequently lead to phenotypic changes between japonica and indica rice in response to biotic stress and had been gradually fixed in population during domestication. 


\section{Conclusions}

In summary, our genome-wide comparative analysis showed that, higher sequence diversity in promoter region is tightly associated with expression divergence of evolutionarily conserved orthologous genes in NPB and 93-11 cultivars, which may subsequently account for their differential response to $M$. oryzae infection. We proposed that variation in the expression pattern of orthologous genes in two rice cultivars may act as an evolutionary alternative distinguishing the two genetically diverse rice subspecies response to $M$. oryzae infection. Genetic variations among the two rice subspecies identified in this study also provide valuable resource that could be explored as the target for genetic engineering research.

\section{Additional file}

Additional file 1: Table S1. Total number of sequencing reads and mapping results for 30 samples in this study. Table S2. qRT-PCR primers for RNA-seq confirmation used in this study. Figure S1. Infection assay of GY11 and FJ87 on NPB and 93-11. Figure S2. Workflow of rice inoculation and samples collections. Figure S3. Pearson Correlation Coefficient between sequenced samples. Figure S4. Correlation $\left(R^{2}\right)$ of 10 DEGs expression between RNA-seq and qRT-PCR results. Figure S5. Expression level of rice defense genes in RNA-seq. Figure S6. Number of conserved orthologous genes in 5 rice genomes. (DOCX $2481 \mathrm{~kb}$ )

\section{Abbreviations}

bp: Base Pair; DEG: Differentially expressed genes; FDR: False discovery rate; FJ87: FJ81278; FPKM: Fragments Per Kilobase of transcript per Million mapped reads; Fst: Fixation index; GO: Gene Ontology; GY11: Guy11; hpi: Hours Post Inoculation; NPB: Nipponbare; nt: Single-stranded DNA/RNA units of nucleotides; qRT-PCR: Quantitative Real Time-PCR; QTL: Quantitative Trait Locus; SNP: Singlenucleotide polymorphism; TF: Transcription factor

\section{Acknowledgements}

We thank members of the Z.W. laboratory for useful discussions.

\section{Funding}

This work was supported by grants from the Natural Science Foundations of China (31770156, U1805232, U1305211, 31401692), the National Key Research and Development Program of China (2016YFD0300700), and the Scientific Research Foundation of the Graduate School of Fujian Agriculture and Forestry University.

\section{Availability of Data and Materials}

The sequencing reads for this study are available in NCBI under BioProject: PRJNA358135.

\section{Authors' Contributions}

$Z Z, J N, H Z$ and $Z W$ designed the research and wrote the manuscript. ZZ, LYL analyzed data. ZZ, XFC, XC, MC, YL and LLL finished inoculation, RNA extraction and GRT-PCR. All authors read and approved the final manuscript.

\section{Ethics Approval and Consent to Participate}

This study complied with the ethical standards of China, where this research work was carried out.

\section{Consent for Publication}

All authors are consent for publication.

\section{Competing Interests}

The authors declare that they have no competing interests.

\section{Publisher's Note}

Springer Nature remains neutral with regard to jurisdictional claims in published maps and institutional affiliations.

\section{Author details}

${ }^{1}$ State Key Laboratory of Ecological Pest Control for Fujian and Taiwan Crops, College of Plant Protection, Fujian Agriculture and Forestry University, Fuzhou 350002, China. ${ }^{2}$ Fujian-Taiwan Joint Center for Ecological Control of Crop Pests, Fujian Agriculture and Forestry University, Fuzhou 350002, China. ${ }^{3}$ College of Life Science, Fujian Agriculture and Forestry University, Fuzhou 350002, China. ${ }^{4}$ Institute of Oceanography, Minjiang University, Fuzhou 350108, China

Received: 31 October 2018 Accepted: 18 February 2019

Published online: 01 March 2019

\section{References}

Arsovski AA, Pradinuk J, Guo XQ, Wang S, Adams KL (2015) Evolution of cisregulatory elements and regulatory networks in duplicated genes of Arabidopsis thaliana. Plant Physiol 169:2982

Ashburner M, Ball CA, Blake JA, Botstein D, Butler H, Cherry JM et al (2000) Gene ontology: tool for the unification of biology. Nat Genet 25:25

Bai X, Huang Y, Hu Y, Liu H, Zhang B, Smaczniak C et al (2017) Duplication of an upstream silencer of FZP increases grain yield in rice. Nat Plants 3:885

Bao J, Chen M, Zhong Z, Tang W, Lin L, Zhang X et al (2017) PacBio sequencing reveals transposable elements as a key contributor to genomic plasticity and virulence variation in Magnaporthe oryzae. Mol Plant 10:1465-1468

Bhuiyan NH, Liu W, Liu G, Selvaraj G, Wei Y, King J (2007) Transcriptional regulation of genes involved in the pathways of biosynthesis and supply of methyl units in response to powdery mildew attack and abiotic stresses in wheat. Plant Mol Biol 64:305-318

Bowles DJ (1990) Defense-related proteins in higher plants. Annu Rev Biochem 59:873-907

Broitman-Maduro G, Maduro MF, Rothman JH (2005) The noncanonical binding site of the MED-1 GATA factor defines differentially regulated target genes in the C. elegans mesendoderm. Dev Cell 8:427-433

Danecek P, Auton A, Abecasis G, Albers CA, Banks E, DePristo MA et al (2011) The variant call format and VCFtools. Bioinformatics 27:2156-2158

Dean R, Van Kan JA, Pretorius ZA, Hammond-Kosack KE, Di Pietro A, Spanu PD et al (2012) The top 10 fungal pathogens in molecular plant pathology. Mol Plant Pathol 13:414-430

Du H, Yu Y, Ma Y, Gao Q, Cao Y, Chen Z et al (2017) Sequencing and de novo assembly of a near complete indica rice genome. Nat Commun 8:15324

Ebbole DJ (2007) Magnaporthe as a model for understanding host-pathogen interactions. Annu Rev Phytopathol 45:437-456

Gallet R, Fontaine C, Bonnot F, Milazzo J, Tertois C, Adreit H et al (2016) Evolution of compatibility range in the rice- magnaporthe oryzae system: an uneven distribution of $R$ genes between rice subspecies. Phytopathology 106:348-354

Goff SA, Ricke D, Lan TH, Presting G, Wang R, Dunn M et al (2002) A draft sequence of the rice genome (Oryza sativa L. ssp. japonica). Science 296:92-100

Haerty W, Singh RS (2006) Gene regulation divergence is a major contributor to the evolution of Dobzhansky-Muller incompatibilities between species of Drosophila. Mol Biol Evol 23:1707-1714

He Z, Zhai W, Wen H, Tang T, Wang Y, Lu X et al (2011) Two evolutionary histories in the genome of rice: the roles of domestication genes. PLoS Genet 7:e1002100

Heinz S, Benner C, Spann N, Bertolino E, Lin YC, Laslo P et al (2010) Simple combinations of lineage-determining transcription factors prime cisregulatory elements required for macrophage and B cell identities. Mol Cell 38:576-589

Hoffmann RD, Olsen LI, Husum JO, Nicolet JS, Thøfner JF, Wätjen AP et al (2016) A cis-regulatory sequence acts as a repressor in the Arabidopsis thaliana sporophyte, but as an activator in pollen. Mol Plant 10;(5):775-778

Huang X, Kurata N, Wei X, Wang ZX, Wang A, Zhao Q et al (2012) A map of rice genome variation reveals the origin of cultivated rice. Nature 490:497-501

Huang X, Sang T, Zhao Q, Feng Q, Zhao Y, Li C et al (2010) Genome-wide association studies of 14 agronomic traits in rice landraces. Nat Genet 42:961

Huang Y, Zhao S, Fu Y, Sun H, Ma X, Tan L et al (2018) Variation in FZP regulatory region causes increases of inflorescence secondary branches and grain yield in rice domestication. Plant J 96(4):716-733 
Jiang SY, Ma Z, Vanitha J, Ramachandran S (2013) Genetic variation and expression diversity between grain and sweet sorghum lines. BMC Genomics 14:18-18

Jung K-H, Dardick C, Bartley LE, Cao P, Phetsom J, Canlas P et al (2008) Refinement of light-responsive transcript lists using rice oligonucleotide arrays: evaluation of gene-redundancy. PLoS One 3:e3337

King MC, Wilson AC (1975) Evolution at two levels in humans and chimpanzees. Science 188:107-116

Konishi S, Izawa T, Lin SY, Ebana K, Fukuta Y, Sasaki T et al (2006) An SNP caused loss of seed shattering during rice domestication. Science 312:1392-1396

Krzywinski M, Schein J, Birol I, Connors J, Gascoyne R, Horsman D et al (2009) Circos: an information aesthetic for comparative genomics. Genome Res 19: 1639-1645

Langmead B, Salzberg SL (2012) Fast gapped-read alignment with bowtie 2. Nat Methods 9:357-359

Li B, Dewey CN (2011) RSEM: accurate transcript quantification from RNA-Seq data with or without a reference genome. BMC Bioinformatics 12:323

Li H, Handsaker B, Wysoker A, Fennell T, Ruan J, Homer N et al (2009) The sequence alignment/map format and SAMtools. Bioinformatics 25:2078-2079

Li M, Chen H, Chen J, Chang M, Palmer IA, Gassmann W et al (2018) TCP transcription factors interact with NPR1 and contribute redundantly to systemic acquired resistance. Front Plant Sci 9:1153

Li W, Zhu Z, Chern M, Yin J, Yang C, Ran L et al (2017) A natural allele of a transcription factor in rice confers broad-spectrum blast resistance. Cell 170: 114-126.e115

Liao J, Huang H, Meusnier I, Adreit H, Ducasse A, Bonnot F et al (2016) Pathogen effectors and plant immunity determine specialization of the blast fungus to rice subspecies. eLife 5:e19377

Liu F, Xu W, Wei Q, Zhang Z, Xing Z, Tan L et al (2010a) Gene expression profiles deciphering rice phenotypic variation between Nipponbare (japonica) and 93-11 (Indica) during oxidative stress. PLoS One 5:e8632

Liu J, Wang X, Mitchell T, Hu Y, Liu X, Dai L et al (2010b) Recent progress and understanding of the molecular mechanisms of the rice-Magnaporthe oryzae interaction. Mol Plant Pathol 11:419-427

Liu M-J, Sugimoto K, Uygun S, Panchy N, Campbell MS, Yandell M et al (2018) Regulatory divergence in wound-responsive gene expression between domesticated and wild tomato. Plant Cell. https://doi.org/10.1105/tpc.18.00194

Liu Y, Ji X, Nie X, Qu M, Zheng L, Tan Z et al (2015) Arabidopsis AtbHLH112 regulates the expression of genes involved in abiotic stress tolerance by binding to their E-box and GCG-box motifs. New Phytol 207:692-709

López-Arredondo D, González-Morales SI, Bello-Bello E, Alejo-Jacuinde G, Herrera $L$ (2015) Engineering food crops to grow in harsh environments. F1000Res 4(F1000 Faculty Rev):651

Lu L, Chen J, Robb SMC, Okumoto Y, Stajich JE, Wessler SR (2017) Tracking the genome-wide outcomes of a transposable element burst over decades of amplification. Proc Natl Acad Sci U S A 114:E10550-E10559

Lu T, Lu G, Fan D, Zhu C, Li W, Zhao Q et al (2010) Function annotation of the rice transcriptome at single-nucleotide resolution by RNA-seq. Genome Res 20:1238-1249

Nawy T, Lee J-Y, Colinas J, Wang JY, Thongrod SC, Malamy JE et al (2005) Transcriptional profile of the Arabidopsis root quiescent center. Plant Cell 17:1908-1925

Nelson DE, Repetti PP, Adams TR, Creelman RA, Wu J, Warner DC et al (2007) Plant nuclear factor Y (NF-Y) B subunits confer drought tolerance and lead to improved corn yields on water-limited acres. Proc Natl Acad Sci 104:16450-16455

Osada N, Kohn MH, Wu C (2006) Genomic inferences of the cis-regulatory nucleotide polymorphisms underlying gene expression differences between Drosophila melanogaster mating races. Mol Biol Evol 23:1585

Palma K, Zhao Q, Cheng YT, Bi D, Monaghan J, Cheng W et al (2007) Regulation of plant innate immunity by three proteins in a complex conserved across the plant and animal kingdoms. Genes Dev 21:1484-1493

Rao PS, Strange RN (1994) Chemistry, biology, and role of groundnut phytoalexins in resistance to fungal attack. In: Daniel M, Purkayastha RP (eds) Handbook of phytoalexin metabolism and action, pp 199-227

Rice P, Longden I, Bleasby A (2000) EMBOSS: the European molecular biology open software suite. Trends Genet 16:276-277

Rifkin SA, Kim J, White KP (2003) Evolution of gene expression in the Drosophila melanogaster subgroup. Nat Genet 33:138-144

Robinson MD, McCarthy DJ, Smyth GK (2010) edgeR: a bioconductor package for differential expression analysis of digital gene expression data. Bioinformatics 26:139-140
Rozas J, Ferrer-Mata A, Sanchez-DelBarrio JC, Guirao-Rico S, Librado P, Ramos Onsins SE et al (2017) DnaSP 6: DNA sequence polymorphism analysis of large data sets. Mol Biol Evol 34:3299-3302

Schaff J, Walter M, Hess D (1995) Primary metabolism in plant defense. Plant Physiol 108:949-960

Siepel A, Arbiza L (2014) Cis-regulatory elements and human evolution. Curr Opin Genet Dev 29:81-89

Sweeney M, McCouch S (2007) The complex history of the domestication of rice. Ann Bot 100:951-957

Tang T, Lu J, Huang J, He J, McCouch SR, Shen Y et al (2006) Genomic variation in rice: genesis of highly polymorphic linkage blocks during domestication. PLoS Genet 2:e199

Tran L-SP, Nakashima K, Sakuma Y, Simpson SD, Fujita Y, Maruyama K et al (2004) Isolation and functional analysis of Arabidopsis stress-inducible NAC transcription factors that bind to a drought-responsive cis-element in the early responsive to dehydration stress 1 promoter. Plant Cell 16:2481-2498

Wang Q, Nian J, Xie X, Yu H, Zhang J, Bai J et al (2018a) Genetic variations in ARE1 mediate grain yield by modulating nitrogen utilization in rice. Nat Commun 9:735

Wang W, Mauleon R, Hu Z, Chebotarov D, Tai S, Wu Z et al (2018b) Genomic variation in 3,010 diverse accessions of Asian cultivated rice. Nature 557:43

Wang X, Li R, Sun C, Li Z, Cai H, Sun X (1998) Identification and classification of subspecies of Asian cultivated rice and their hybrids. Chin Sci Bull 43:1864-1872

Wang Y, Tang H, DeBarry JD, Tan X, Li J, Wang X et al (2012) MCScanX: a toolkit for detection and evolutionary analysis of gene synteny and collinearity. Nucleic Acids Res 40:e49

Wildermuth MC, Dewdney J, Wu G, Ausubel FM (2001) Isochorismate synthase is required to synthesize salicylic acid for plant defence. Nature 414:562

Wray GA (2007) The evolutionary significance of cis-regulatory mutations. Nat Rev Genet 8:206-216

Xie W, Wang G, Yuan M, Yao W, Lyu K, Zhao H et al (2015) Breeding signatures of rice improvement revealed by a genomic variation map from a large germplasm collection. Proc Natl Acad Sci 112(39):E5411-E5419

Xing YZ, Tang WJ, Xue WY, Xu CG, Zhang Q (2008) Fine mapping of a major quantitative trait loci, qSSP7, controlling the number of spikelets per panicle as a single Mendelian factor in rice. Theor Appl Genet 116:789-796

Yang H, Ludewig U (2014) Lysine catabolism, amino acid transport, and systemic acquired resistance: what is the link? Plant Signal Behav 9:e28933

Yeaman S, Hodgins KA, Suren H, Nurkowski KA, Rieseberg LH, Holliday JA et al (2014) Conservation and divergence of gene expression plasticity following c. 140 million years of evolution in lodgepole pine (Pinus contorta) and interior spruce (Picea glaucax Picea engelmannii). New Phytol 203:578-591

Yoshida H, Haze K, Yanagi H, Yura T, Mori K (1998) Identification of the cis-acting endoplasmic reticulum stress response element responsible for transcriptional induction of mammalian glucose-regulated proteins involvement of basic leucine zipper transcription factors. J Biol Chem 273: 33741-33749

Yu J, Hu S, Wang J, Wong GK, Li S, Liu B et al (2002) A draft sequence of the rice genome (Oryza sativa L. ssp. indica). Science 296:79-92

Zeier J (2013) New insights into the regulation of plant immunity by amino acid metabolic pathways. Plant Cell Environ 36:2085-2103

Zhang J, Chen LL, Xing F, Kudrna DA, Yao W, Copetti D et al (2016) Extensive sequence divergence between the reference genomes of two elite indica rice varieties Zhenshan 97 and Minghui 63. Proc Natl Acad Sci U S A 113: E5163-E5171

Zhong Z, Chen M, Lin L, Han Y, Bao J, Tang W et al (2018) Population genomic analysis of the rice blast fungus reveals specific events associated with expansion of three main clades. ISME J 12(8):1867-1878 